\title{
A Face Recognition System for Assistive Robots
}

\author{
Samuel-Felipe Baltanas, Jose-Raul Ruiz-Sarmiento, Javier Gonzalez-Jimenez \\ Machine Perception and Intelligent Robotics Group (MAPIR), \\ System Engineering and Automation Department, \\ Biomedical Research Institute of Málaga (IBIMA), \\ University of Málaga, Campus de Teatinos, 29071, Malaga, Spain.
}

\begin{abstract}
Assistive robots collaborating with people demand strong Human-Robot interaction capabilities. In this way, recognizing the person the robot has to interact with is paramount to provide a personalized service and reach a satisfactory end-user experience. To this end, face recognition: a non-intrusive, automatic mechanism of identification using biometric identifiers from an user's face, has gained relevance in the recent years, as the advances in machine learning and the creation of huge public datasets have considerably improved the state-of-the-art performance. In this work we study different open-source implementations of the typical components of state-of-the-art face recognition pipelines, including face detection, feature extraction and classification, and propose a recognition system integrating the most suitable methods for their utilization in assistant robots. Concretely, for face detection we have considered MTCNN, OpenCV's DNN, and OpenPose, while for feature extraction we have analyzed InsightFace and Facenet. We have made public an implementation of the proposed recognition framework, ready to be used by any robot running the Robot Operating System (ROS). The methods in the spotlight have been compared in terms of accuracy and performance in common benchmark datasets, namely FDDB and LFW, to aid the choice of the final system implementation, which has been tested in a real robotic platform.
\end{abstract}

Published in: International Conference on Applications of Intelligent Systems (APPIS), Las Palmas de Gran Canaria (Spain), 2020.

DOI: https://doi.org/10.1145/3378184.3378225

More Info: http://mapir.uma.es/mapirwebsite/index.php/mapir-downloads/papers/XXX

Cite this paper as: S.F. Baltanas-Molero, J.R. Ruiz-Sarmiento, J. Gonzalez-Jimenez, "A Face Recognition System for Assistive Robots", International Conference on Applications of Intelligent Systems (APPIS), Las Palmas de Gran Canaria (Spain), 2020. 